\title{
Use of Suprathel® as a complete epidermal substitute in a boy with extensive toxic epidermal necrolysis
}

\author{
${ }^{1}$ Renkert-Baudis M, ${ }^{2}$ Schöler M, ${ }^{3}$ Demirakca S, 3Jung T, ${ }^{4}$ Mockenhaupt M, ${ }^{1}$ Lange B \\ 1Department of Pediatric Surgery, Pediatric Burn Center, University Medical Center, 68167 Mannheim, Germany \\ 2Department of Anaesthesiology and Surgical Intensive Care, University Medical Center, 68167 Mannheim, Germany \\ ${ }^{3}$ Department of Neonatology and Pediatric Intensive Care, University Medical Center, 68167 Mannheim, Germany \\ ${ }^{4}$ Department of Dermatology, University Freiburg - Medical Center, 79104 Freiburg, Germany
}

\section{Objectives}

Toxic epidermal necrolysis (TEN) is a rare, potentially life-threatening condition characterized by extensive loss of skin and mucosa of more than $30 \%$ total body surface area (TBSA). It is linked to certain drugs as well as viral or bacterial infections, resulting in a dysregulated immune reaction against epithelial cells. Treatment, as in thermal injuries, should include an early referral to a burn unit. This case report demonstrates a life-saving management of TEN with an epidermal substitute (Suprathel ${ }^{\circledR}$ ) in a pediatric patient with epidermal damage of $100 \%$ TBSA.

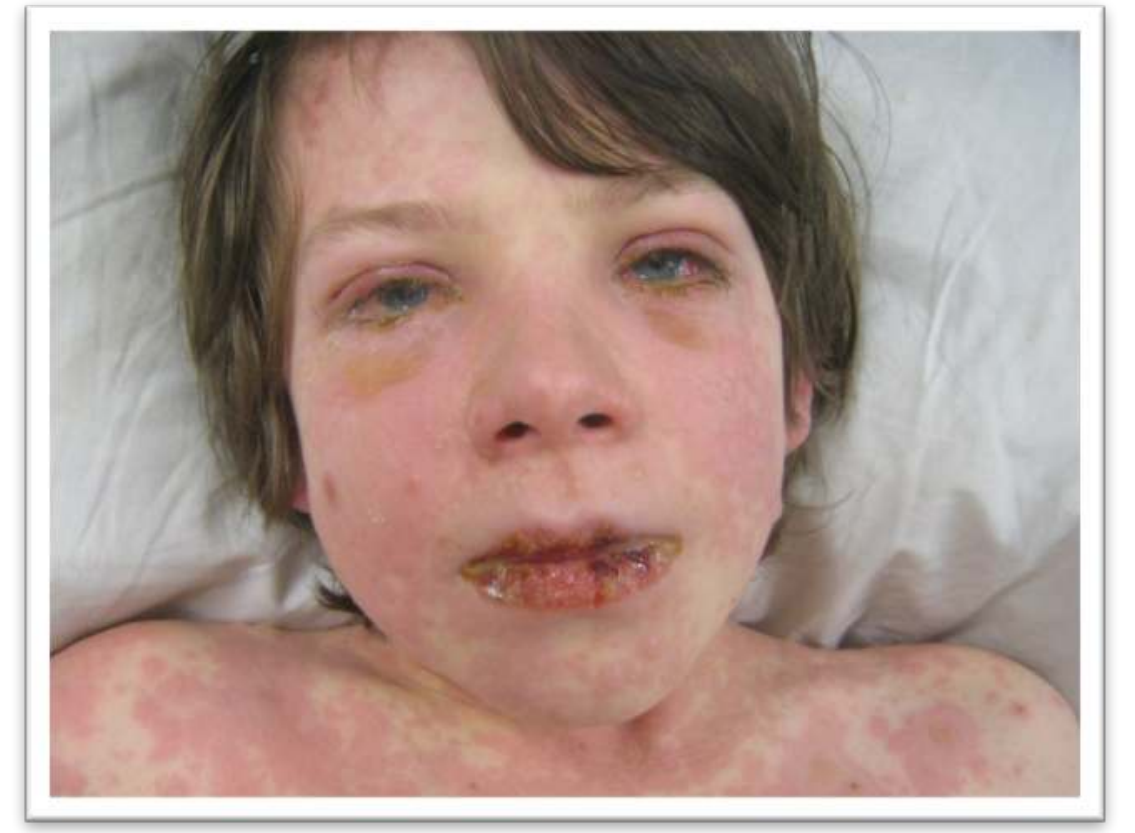

Clinical presentation on admission (after flu-like prodromal stadium): severe conjunctivitis, mucosal erosions, macules

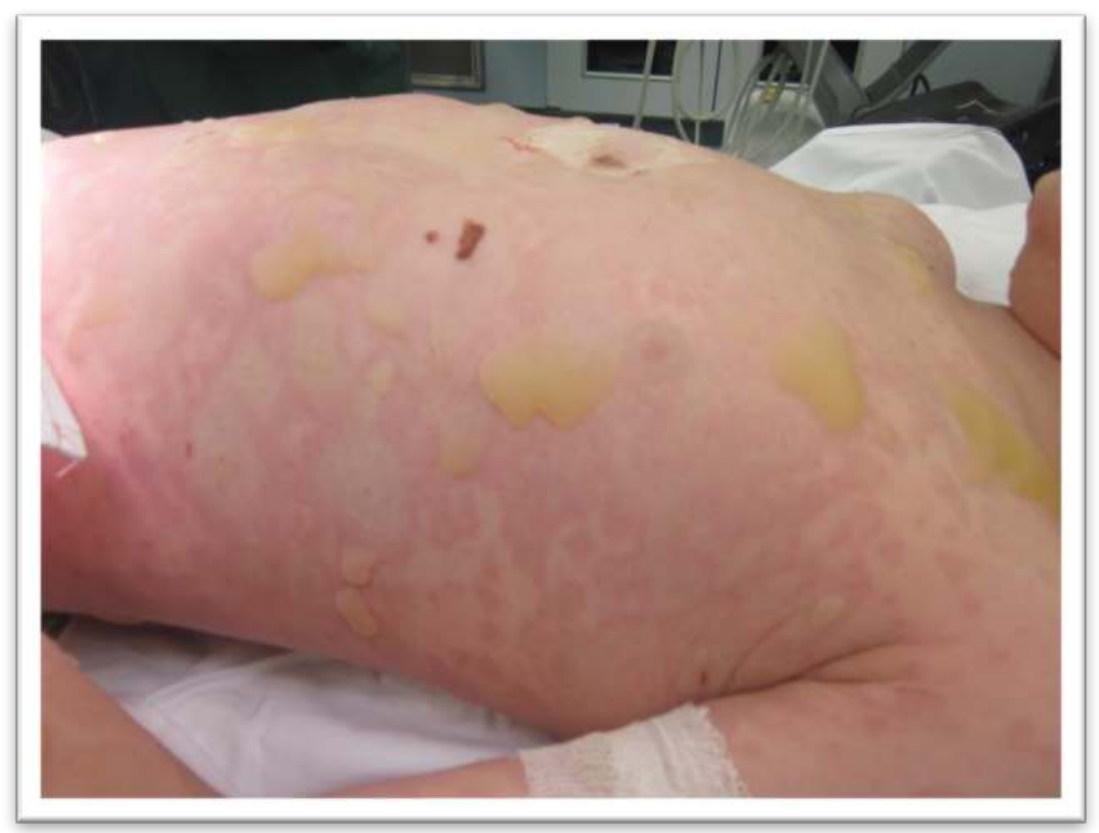

Epidermal necrolysis, 100\% TBSA

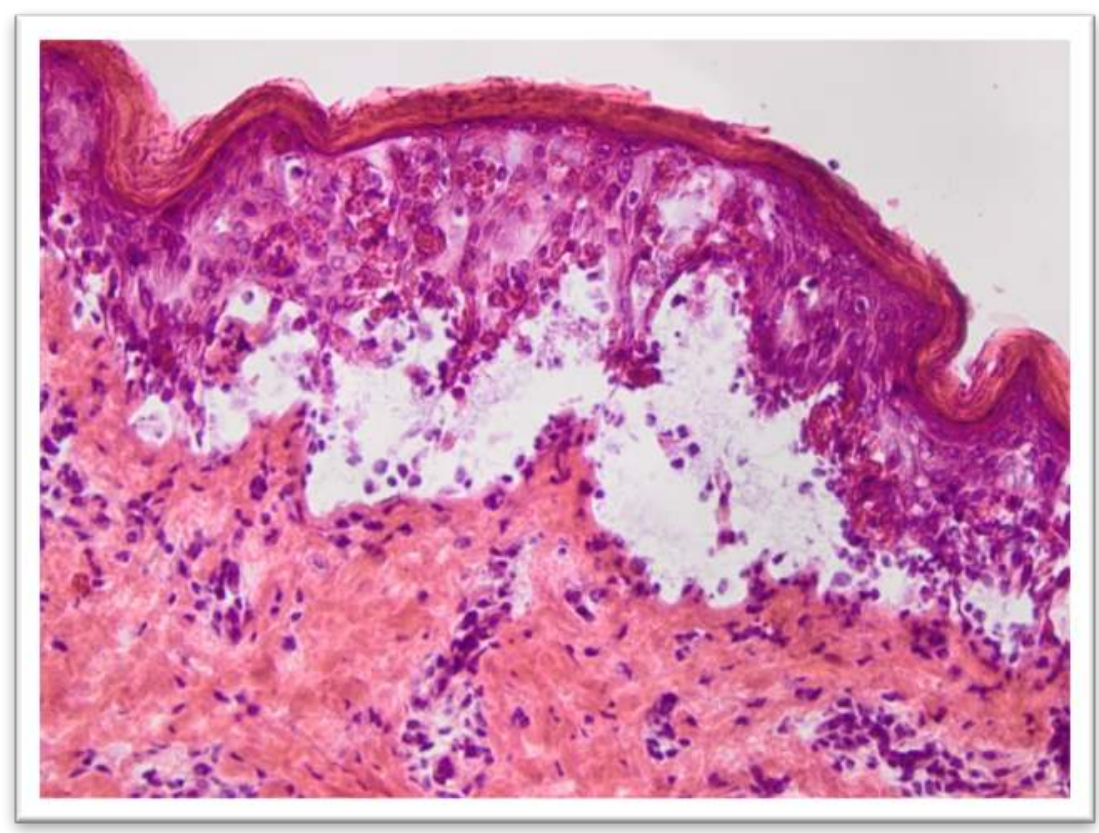

Skin biopsy with complete detachment of the epidermal layer

\section{Methods}

A ten-year-old boy was admitted to our pediatric burn center complaining of foreign body sensation and photosensitivity of the eyes, headaches, pyrexia and presenting with extensive epidermolysis involving 100\% TBSA. A skin biopsy confirmed the diagnosis of TEN with a complete loss of the epidermal layer as well as extensive mucosal involvement. The patient required fluid resuscitation and admission to the pediatric intensive care unit. Wound care consisted of gentle debridement of the blistered areas followed by extensive Suprathel® application.

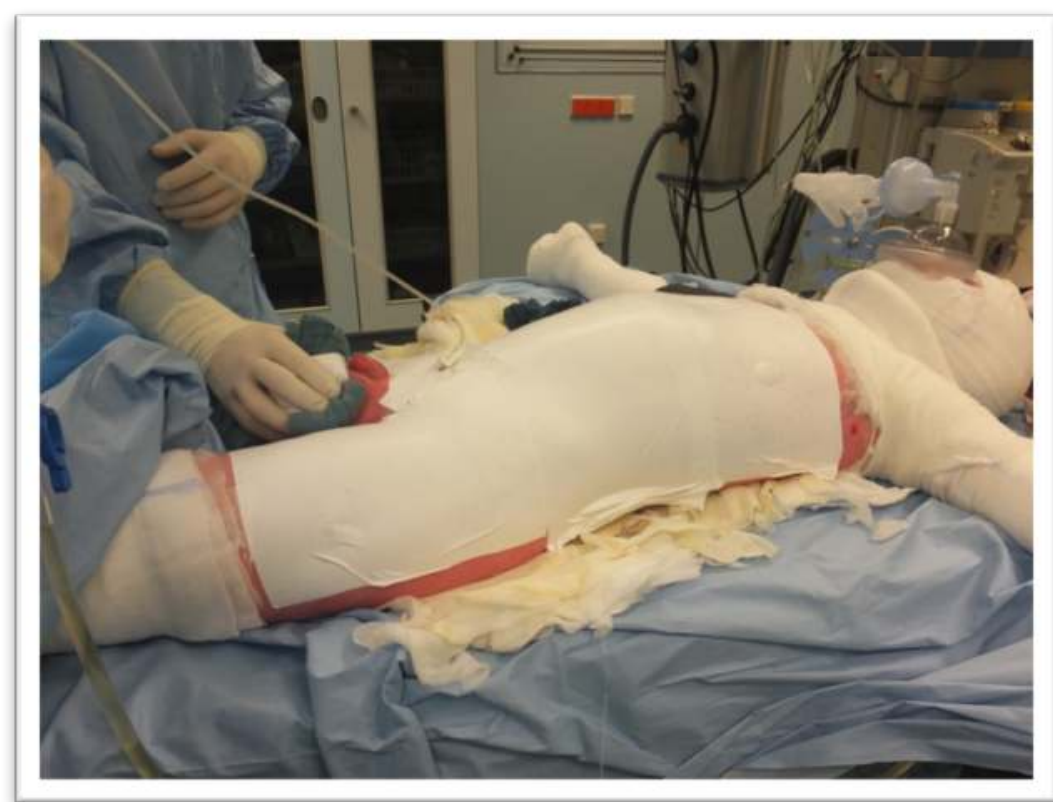

Suprathel® application after debridement, nonintubated spontaneously breathing patient

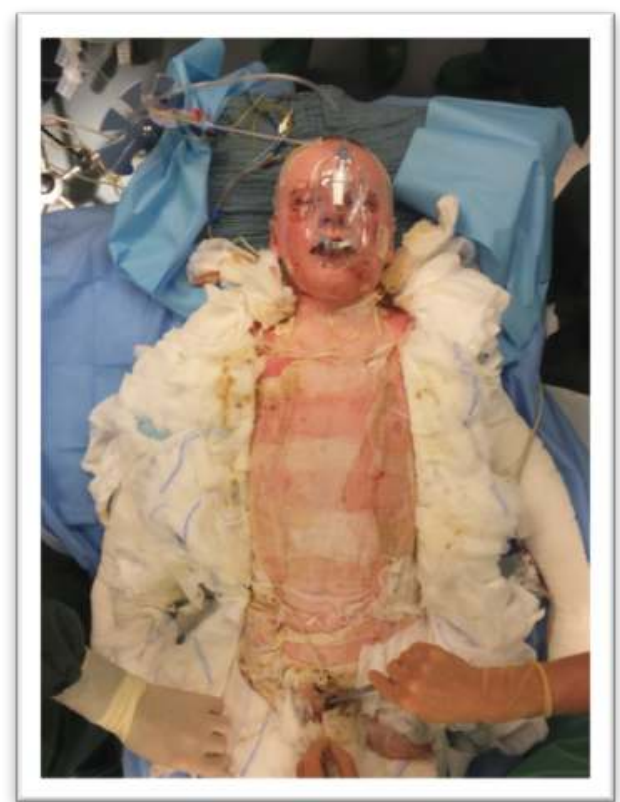

Dressing change under sedation
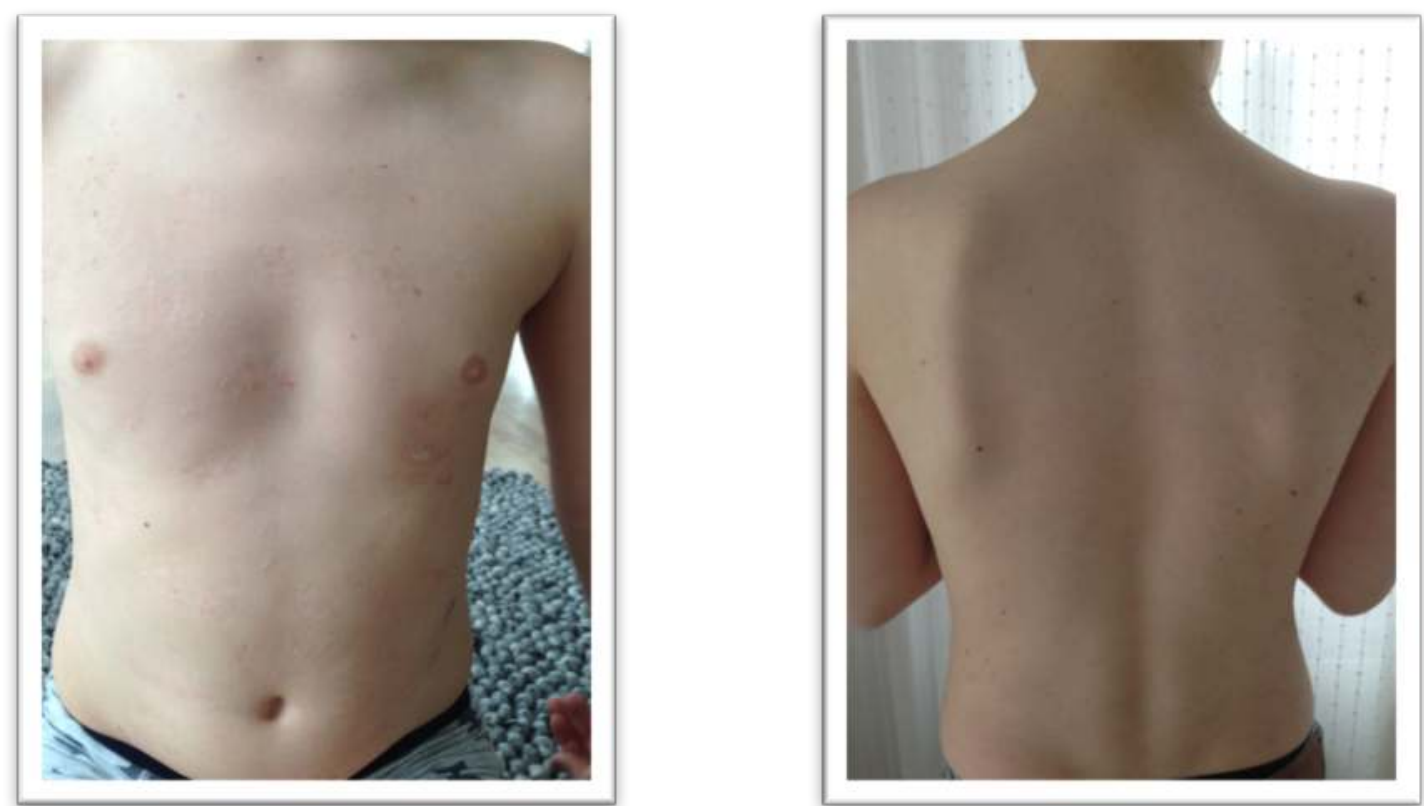

Two years after trauma: no scarring, some areas of hypopigmentation, full range of motion, unimpaired vision

\section{Results}

Repeated Suprathel® application maintained the skin barrier function over time and resulted in almost complete reepithelialization. The mucosa of the urogenital tract and eyes showed an almost complete restitution, as well. After a six-week inpatient stay the patient could be discharged to our outpatient care.

Two years after treatment the patient is satisfied with the results and the skin is virtually free of scars with a normal appearance and elasticity.

\section{Conclusion}

In children, TEN with extensive epidermal loss is a rare, life-threatening condition that requires admission to a specialized pediatric burn unit. Suprathel ${ }^{\circledR}$ proves beneficial in the management of these cases by providing a skin barrier until natural reepithelialization occurs. 